\title{
Visfatin in Association with Severity and Activity of Primary Osteoarthritis and Rheumatoid Arthritis
}

\author{
Dalia Allam, Abd El Wahab Shams El Din, Ahmed El Shambaky, Marwa Yehia
}

\begin{abstract}
Department of Rheumatology, Rehabilitation and physical medicine, Benha faculty of medicine , Benha University, Egypt.
\end{abstract}

Correspondence to: Dalia Allam, Department of Rheumatology, Rehabilitation and physical medicine, Benha faculty of medicine, Benha University, Egypt.

Email:

dalia.allam1987@yahoo.com

Received: 4 October 2020

Accepted: 13 December 2020

\begin{abstract}
:
Background: Visfatin is a one of the recently discovered adipokine that has important pro-inflammatory and catabolic roles in rheumatoid arthritis (RA). Pro-inflammatory cytokines initiate a number of physiological changes that result in the characteristic signs of inflammation. Visfatin may play a role in OA by activating chondrocytes. Along with its cytokine effect, visfatin has an enzymatic activity called nicotinamide-phosphoribosyl-transferase (NAMPT). Objectives: To study the level of Visfatin in RA and OA sera and correlate its levels with activity and severity scores of both diseases. Subjects and methods: A case control study was conducted to assess Visfatin serum level in 20 patients of osteoarthritis and 25 patients rheumatoid arthritis and 20 apparently healthy individuals. Lequesne's algo-functional Index used for assessment of OA functional impairment, Kellgren and Lawrence for radiological severity and KOFUS for activity, while Larsen's
\end{abstract} scoring system used for assessment of RA severity and DAS 28 score for activity. Visfatin concentrations in serum measured using a commercially available ELISA kit. Results: Mean Lequesne's index was $11.2 \pm$ 3.6. According to Kellgren and Lawrence we had one patient G1 , 9 patients GII , 7 patients GIII and 3 patients GIV .Median KOFUS was 5 and it ranged from 3 to 13, Mean DAS 28 was $4.96 \pm 1.27$. As regards Larsen score, mean was $24 \pm 22$. There was an overall significance in visfatin between three groups. $\mathrm{P}$ value was $<0.001$. Pairwise comparisons showed that mean visfatin was significantly higher in group I (10.68) compared to group III (5.3) and it was significantly higher in group II (14.26) compared to group III (5.3). There was a significant positive correlation between visfatin and Lequesne's index. $(\mathrm{r}=0.731 \& \mathrm{P}$ value $<0.001)$. There was a significant positive correlation between visfatin and DAS $28(\mathrm{r}=0.618 \& \mathrm{P}$ 
value $=0.001)$. Conclusion: Visfatin may have a role in the pathogenesis of OA and RA. Furthermore it acts as a potential marker for disease activity and severity of RA as well as severity of OA.

Keywords: Visfatin, Rheumatoid arthritis(RA), Osteoarthritis (OA), ELISA kit.

\section{Introduction:}

Osteoarthritis (OA) is the most prevalent articular disease, and the most common cause of disability in developed and developing countries ,with knee involvement being the most prevalent and disabling condition.[1]

Among the soluble mediators released by synovium potentially involved in $\mathrm{OA}$ pathophysiology, the so-called adipokines, these mediators are secreted mainly from adipose tissue and found in the biological fluids and may participate in synoviumbone and synovium-cartilage interactions.[2]

Interest is growing in the adipokine visfatin, also called pre-B-cell colony-enhancing factor or nicotinamide-phosphoribosyltransferase.[3]

The exact role of adipokines in KOA is not well known, but they may play a significant role indirectly via their link to obesity and directly on OA pathways in many patients. Researchers in different studies, have evaluated the relationship between adipokines individually or controlled by some metabolic, other cytokines and clinical severity [4].

$\mathrm{RA}$ is an autoimmune multisystem inflammatory disease characterized mainly by articular synovitis leading to arthritis and stiffness. [5]

The diagnosis of RA depends on combination of manifestation, laboratory investigations as well as imaging. Several autoantibodies were developed to get early diagnosis and predict prognosis.[5]

Visfatin induces the production of interleukin (IL-1b), tumor necrosis factor-a (TNF-a), and IL-6. Moreover, it increases the surface expression of co-stimulatory molecules CD54, CD40, and CD80. It enhances B-cell differentiation, initiation of cytokines and matrix metalloproteinases, and inhibits neutrophil apoptosis, thus playing a key role in the persistence of inflammation in RA [5]. 
The purpose of this study was to study the visfatin level and its relation to the disease activity and severity in RA and OA diseases.

\section{Subjects and methods:}

A case control study included 45 patients and 20 apparently healthy individuals. All patients were attending inpatient and outpatient clinics of Rheumatology, Rehabilitation, and Physical Medicine Department, Benha University Hospitals starting from May 2019 to February 2020.

The study was accepted by Benha Faculty of Medicine Ethical Committee No. 2017

The subjects were grouped as:

Group I: Twenty (20) patients with primary knee osteoarthritis (OA).We selected knee OA as a model for primary OA, so the patients could have $\mathrm{OA}$ in other joints like hand and hip but we chosen knee OA for easier screening by radiology and for the availability of Arabic translated questionnaire used for evaluation.

Group II: Twenty-five (25) patients with Rheumatoid Arthritis (RA).

Group III: Twenty (20) apparently healthy individuals, age and sex matched to RA and OA patients as controls.
Patients of OA in this study met the American College of Rheumatology (ACR) clinical/radiographic symptomatic criteria osteoarthritis. [6].Patients of RA in this study met the 2010 ACR /EULAR RA classification criteria [7].

An Arabic written consent was taken from all participants.

All patients were subjected to: full history taking (personal, complaint, present, past, and family history), full clinical examination, laboratory assessment: Complete blood picture (CBC), Erythrocyte sedimentation rate (ESR), $\mathrm{C}$ reactive protein (CRP), Rheumatoid factor titter (RF) .

Assessment of disease activity of RA by (DAS-28) and severity by Larsen's scoring system. OA severity (functional impairment) by Lequesne's algo-functional Index , radiological by Kellgren and Lawrence while OA activity by KOFUS .

Measurement of serum visfatin level using a double-antibody sandwich ELISA (Enzyme Linked Immune Sorbent Assay) to detect serum level of Nicotinamide phosphoribosyl transferase (NAMPT) or visfatin using a commercial Human (NAMPT) ELISA Kit. (Cat \#: 201-12-8417, SunRedBio, China). 



\section{Statistical analysis:}

Data management and statistical analysis were done using SPSS vs.25. (IBM, Armonk, New York, United states).

Due to small number of patient samples we used non-parametrical distribution to test normality to be more conservative. Categorical data was summarized as numbers and percentages.

Comparisons between groups were done using Kruskal Wallis test for numerical data. Categorical data was compared using Fisher's exact test if appropriate. In case of overall significance, post hoc analysis was done and all post hoc comparisons were Bonferroni adjusted. ROC analysis was done for vistafin in diagnosing osteoarthritis and rheumatoid arthritis. Area Under Curve (AUC) with $95 \%$ confidence interval, best cutoff point and diagnostic indices were calculated.

\section{Results:}

The current study involved 18 females (90\%) and 2 males (10\%) with OA, 24 females $(96 \%)$ and 1 male (4\%) with RA beside 20 volunteers as a control group.
The subjects were selected age and sex matched.The clinical and laboratory characteristics of three groups shared in (table 1)

As regards the Visfatin level, there was a statistically significant difference between the three groups ( $\mathrm{P}$ value $<0.001)$. Pairwise a comparison revealed a statistically significant difference between $\mathrm{OA}$ and controls as well as RA and controls while no significant difference between $\mathrm{OA}$ and RA groups was statistically noticed (Table 2)

In OA group the functional impairment was evaluated by Lequesne's score revealing 5 patients with moderate grade, 5 with severe grade , 4 with very severe grade and 5 with extremely severe grade of functional impairment(Mean Lequesne's score 11.2 $\pm 3.6)$. Parallelly, the radiological grading by Kellgren \& Lawrence determined $5 \%$ of OA patients in grade I, $45 \%$ in grade II, $35 \%$ in grade III and $15 \%$ in grade IV. Sixty percent of the patients had swollen knee joints.

The flare up was evaluated by KOFUS and 9 patients were in activity with median KOFUS 5 (range 3-13). 

Table (1) laboratory tests in the three groups:

\begin{tabular}{|c|c|c|c|c|}
\hline \multicolumn{2}{|c|}{ Laboratory data } & \multirow{2}{*}{$\begin{array}{l}\text { Range } \\
9.5-13\end{array}$} & \multirow{2}{*}{$\begin{array}{l}\text { Mean } \pm \text { S. D } \\
11.3 \pm 1\end{array}$} & \multirow[t]{2}{*}{ p. value } \\
\hline$\overline{\mathrm{HB}}$ & GI OA & & & \\
\hline \multirow[t]{2}{*}{$(\mathrm{g} / \mathrm{dl})$} & GII RA & $8.5-13.5$ & $11 \pm 1.6$ & 0.478 \\
\hline & GIII control & $9.8-12.8$ & $11 \pm 1.2$ & \\
\hline \multirow[t]{3}{*}{ WBCs X10 $/ \mathrm{ml}$} & GI OA & $4.2-9.1$ & $7.21 \pm 1$ & \\
\hline & GII RA & $5-9$ & $7.41 \pm 1.17$ & 0.077 \\
\hline & GIII control & $4.5-9.5$ & $6.79 \pm 1.21$ & \\
\hline Platelets & GI OA & $200-272$ & $239 \pm 19$ & \\
\hline \multirow[t]{2}{*}{ x $10^{6} / \mathrm{ml}$} & GII RA & $204-546$ & $283 \pm 74$ & 0.007 \\
\hline & GIII control & $200-245$ & $228 \pm 16$ & \\
\hline \multirow[t]{3}{*}{ FBS (mg/dl) } & GI OA & $74-225$ & $102 \pm 32$ & \\
\hline & GII RA & $75-243$ & $101 \pm 36$ & 0.099 \\
\hline & GIII control & $72-100$ & $89 \pm 7$ & \\
\hline ALT & GI OA & $12.4-35$ & $23.5 \pm 3.2$ & \\
\hline \multirow[t]{2}{*}{$(\mathbf{U} / \mathbf{L})$} & GII RA & $13.3-40$ & $25.5 \pm 5.7$ & 0.051 \\
\hline & GIII control & $12.5-28$ & $22.1 \pm 3.2$ & \\
\hline AST & GI OA & $13-40$ & $26.23 \pm 4.23$ & \\
\hline \multirow[t]{2}{*}{$(\mathbf{U} / \mathbf{L})$} & GII RA & $20-45$ & $29.33 \pm 6.94$ & 0.095 \\
\hline & GIII control & $10-35$ & $24.23 \pm 6.62$ & \\
\hline \multirow[t]{3}{*}{ Bl. Urea (mg/dl) } & GI OA & $7-19$ & $13.64 \pm 3.1$ & \\
\hline & GII RA & $8-19$ & $13.65 \pm 3.3$ & 0.997 \\
\hline & GIII control & $7-19$ & $13.6 \pm 3.4$ & \\
\hline \multirow{3}{*}{$\begin{array}{l}\text { Creatinine } \\
(\mathrm{mg} / \mathrm{dl})\end{array}$} & GI OA & $0.54-1.1$ & $0.98 \pm 0.3$ & \\
\hline & GII RA & $0.56-1.3$ & $0.94 \pm 0.25$ & 0.085 \\
\hline & GIII control & $0.48-1.1$ & $0.95 \pm 0.1$ & \\
\hline \multirow{3}{*}{$\begin{array}{l}\text { S. bilirubin } \\
\text { (mg/dl) }\end{array}$} & GI OA & $0.2-1.5$ & $0.70 \pm 0.35$ & \\
\hline & GII RA & $0.2-1.1$ & $0.68 \pm 0.31$ & 0.798 \\
\hline & GIII control & $0.2-2.2$ & $0.71 \pm 0.40$ & \\
\hline \multirow{3}{*}{$\begin{array}{c}\text { Prothrombin } \\
\text { concentration \% }\end{array}$} & GI OA & $80-100$ & $93.90 \pm 6.25$ & \\
\hline & GII RA & $80-100$ & $91.90 \pm 7.25$ & 0.101 \\
\hline & GIII control & $80-100$ & $98.2 \pm 10.95$ & \\
\hline \multirow[t]{3}{*}{ Uric acid (mg/dl) } & GI OA & $2.6-5.6$ & $4.35 \pm 0.79$ & \\
\hline & GII RA & $3.1-5.7$ & $3.45 \pm 0.69$ & 0.085 \\
\hline & GIII control & $2.9-5.8$ & $4.23 \pm 0.51$ & \\
\hline
\end{tabular}

P. value : Probability value, SD: Standard deviation. $\mathrm{P}<0.05=$ significant, $\mathrm{P}>.05=$ insignificant, $\mathrm{P}=0.001=$ high significant., WBCs: White blood cells, PLT: Platelet count ,ALT :alanine transaminase test ,AST: Aspartate Aminotransferase test.

Table (2) Vistafin level in study groups

\begin{tabular}{llcccc}
\hline & $\begin{array}{c}\text { Group I } \\
(\mathbf{n}=\mathbf{2 0})\end{array}$ & $\begin{array}{c}\text { Group II } \\
(\mathbf{n}=\mathbf{2 5})\end{array}$ & $\begin{array}{c}\text { Group III } \\
(\mathbf{n}=\mathbf{2 0})\end{array}$ & P value \\
\hline Visfatin & Mean \pm SD & $10.68 \pm 6.52 \mathrm{a}$ & $14.26 \pm 6.79 \mathrm{a}$ & $5.3 \pm 3.98 \mathrm{~b}$ & $<0.001$ \\
\hline
\end{tabular}

Kruskal Wallis test was used. Post-hoc analysis was done \& different letters indicate significant pair, similar letters mean non- significant pair 

ROC analysis was done for visfatin in diagnosis of osteoarthritis. It showed Area Under Curve of $72.5 \%$ with $95 \%$ confidence interval ranging from $55.5 \%$ to $89.5 \%$. Best cutoff was $5.7 \mathrm{ng} / \mathrm{ml}$ at which sensitivity and specificity were $75.0 \% \quad \& \quad 80.0 \%$ respectively. $\mathrm{P}$ value was 0.015. (Fig.1) There was a significant positive correlation between visfatin and Lequesne's index. ( $\mathrm{r}=$ $0.731 \& \mathrm{P}$ value $<0.001)$ and Kellgren \& Lawrence score $(r=0.715 \& \mathrm{P}$ value 0.001).There were no significant correlations between visfatin and other parameters in group I. Table (3)

Logistic regression was done for prediction of osteoarthritis. It was found that Vistafin was a significant predictor with $\mathrm{OR}=1.22$ and $95 \%$ confidence interval ranging from 1.047 to 1.424 . $\mathrm{P}$ value was 0.011 .

In RA group, the activity was evaluated by DAS 28 score revealing four patients with low activity, nine with moderate activity and twelve with high activity (mean DAS 28
$4.96 \pm 1.27)$. While severity were evaluated by Larsen's score revealing 5 patients were low grade, 9 were moderate grade and 11 were high grade (mean Larsen’s 24 \pm 22 )

ROC analysis was done for visfatin in diagnosis of Rheumatoid arthritis. It showed AUC of $87.0 \%$ with $95 \%$ confidence interval ranging from $76.5 \%$ to $97.5 \%$. Best cutoff was $6.8 \mathrm{ng} / \mathrm{ml}$ at which sensitivity and specificity were $76.0 \% \& 85.0 \%$ respectively. $\mathrm{P}$ value was $<0.001$. (Fig. 2)

There was a significant positive correlation between visfatin and DAS $28(r=0.618 \& \mathrm{P}$ value $=0.001)$. There were no significant correlations between visfatin and other parameters in group II.

Logistic regression was done for prediction of rheumatoid arthritis. It was found that Vistafin was a significant predictor with $O R$ $=1.273$ and $95 \%$ confidence interval ranging from 1.11 to 1.461 . P value was 0.001 . 



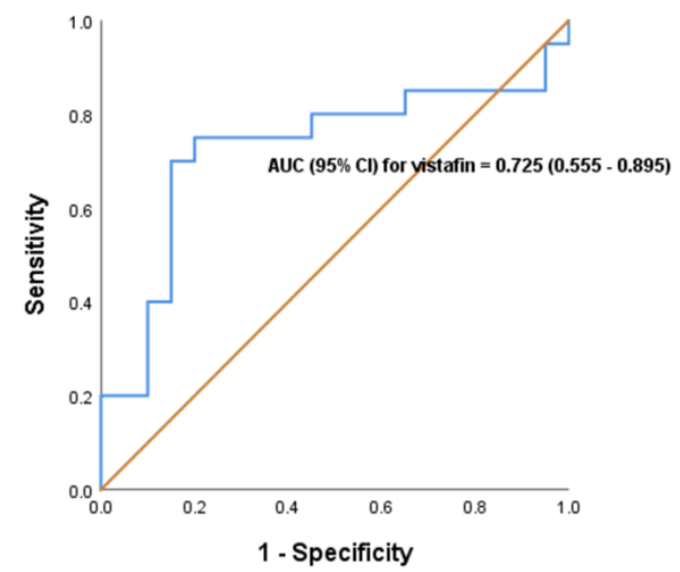

Figure 1: ROC analysis for vistafin in Rheumatoid arthritis

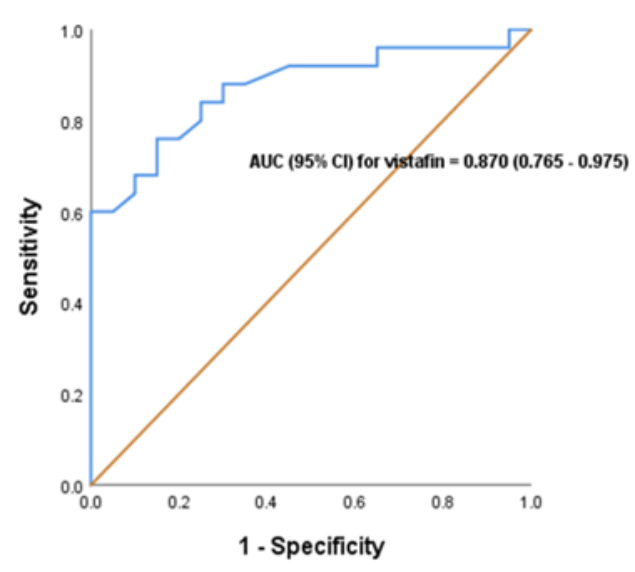

Figure 2: ROC analysis for vistafin in diagnosis of diagnosis of osteoarthritis

Table (3): Correlation between vistafin and other parameters in group I. $r=$ Correlation coefficient

\begin{tabular}{lll}
\hline & Visfatin & \\
& $\mathrm{r}$ & P value \\
\hline Age & 0.14 & 0.556 \\
Lequesne's Index & 0.731 & $<0.001$ \\
Kellgren \& Lawrence & 0.715 & 0.001 \\
KOFUS & 0.396 & 0.084 \\
Morning stiff. Minutes & 0.205 & 0.387 \\
ESR (mm/h) & -0.253 & 0.281 \\
CRP (mg/l) & -0.206 & 0.383 \\
HB (g/dl) & 0.134 & 0.573 \\
Plat X10 $3 / \mathrm{ml}$ & 0.389 & 0.09 \\
Uric acid (mg/dl) & 0.22 & 0.352 \\
\hline
\end{tabular}


Table (4) Correlation between vistafin and other parameters in group II

\begin{tabular}{lcc}
\hline & \multicolumn{2}{c}{ Visfatin } \\
& $\mathrm{r}$ & P value \\
\hline Age & -0.041 & 0.847 \\
Morning stiff. Minutes & 0.132 & 0.529 \\
DAS28 & 0.618 & 0.001 \\
Larsen & 0.176 & 0.399 \\
ESR $(\mathrm{mm} / \mathrm{h})$ & 0.338 & 0.098 \\
CRP $(\mathrm{mg} / \mathrm{l})$ & -0.072 & 0.731 \\
HB $(\mathrm{g} / \mathrm{dl})$ & -0.115 & 0.585 \\
Plat $\left(\mathrm{X} 10^{3} / \mathrm{ml}\right)$ & 0.163 & 0.437 \\
RF $(\mathrm{IU} / \mathrm{ml})$ & 0.211 & 0.311 \\
FBS $(\mathrm{mg} / \mathrm{dl})$ & 0.24 & 0.248 \\
ALT $(\mathrm{U} / \mathrm{mL})$ & 0.168 & 0.422 \\
Creatinine $(\mathrm{mg} / \mathrm{dl})$ & -0.311 & 0.13 \\
\hline
\end{tabular}

\section{Discussion:}

$\mathrm{RA}$ is the most common autoimmune chronic inflammatory arthritis, whose main characteristic is persistent joint inflammation that results in joint damage and loss of function [8]. Early diagnosis of RA is a major challenge for clinical rheumatologists. This is because there is considerable evidence that early treatment with disease modifying antirheumatic drugs leads to a better disease outcome. As current predictors of joint destruction in RA have low specificity, serological biomarkers reflecting bone and cartilage destruction have been proposed as tools in assessing the prognosis of this disease [9]. High levels of visfatin in plasma and synovial fluid have been found in RA patients. In the last few years, visfatin has generated much interest concerning its role during the development of RA [10].

Osteoarthritis is a multifactorial disease. In particular, obesity and increased BMI have been identified as important risk factors. Since mechanical stress cannot explain such a correlation, several systemic factors, referred to as adipokine, have been proposed. Visfatin, an adipokine expressed by fat cells 
has been shown to play a key role in the persistence of inflammation. According to other studies, high serum visfatin concentrations found in patients with arthritis may be related to modulation of the inflammatory response [4].

In our study we measured visfatin serum level in patients of RA and OA as a part of their pathogenesis and demonstrated the relation between its level and each disease activity and severity.

This case control study was carried out on 25 RA patients (Mean age $52 \pm 3$ ). 96\% were females and 4\% were males, $20 \mathrm{OA}$ patients (Mean age $55 \pm 4,90 \%$ were females and $10 \%$ were males).

In RA group, there was higher visfatin level in patients' sera than in control group and there was statistically significant difference between visfatin levels of both groups (14.26 $\mathrm{ng} / \mathrm{ml} \& 5.3 \mathrm{ng} / \mathrm{ml}$ respectively).

Alkady et al. [11] showed that visfatin levels correlated with disease activity and may be involved in the progression of RA.

El-Hini et al. [12] demonstrated positive and significant correlation between visfatin and insulin resistance and also with serum cholesterol, low density lipoprotein cholesterol (LDL-c) and triglycerides.
In a prospective study on 40 patients with RA and 30 controls evaluating visfatin level and its relationship with disease activity and serum lipids Sglunda et al.,[13] found a positive correlation between visfatin levels and disease activity and they demonstrated reduced levels after treatment.[13]

In our study, serum visfatin levels in RA patients were significantly higher than controls, and were significantly higher in severe RA group than moderate RA group which was significantly higher than mild RA group.

These findings go in hand with Elis Carolina et.al. [9], who made a Systematic Reviews and Meta-Analyses (PRISMA) on clinical studies, controlled clinical trials, meta-analysis, multicentric, observational studies, and randomized controlled trials on level of Visfatin in patients with RA, and found patients with RA showed considerably higher plasma levels of visfatin than healthy controls.

In Osteoarthritis group there was a significant positive correlation between visfatin and Lequesne's index. $(\mathrm{r}=0.731 \& \mathrm{P}$ value $<0.001)$. There were no significant correlations between visfatin and other parameters. 
Joan Calvet et.al, [14] cross-sectional study included female patients with symptomatic primary knee osteoarthritis. The disease severity was evaluated according to Kellgren-Lawrence scale and Lequesne index .They measured 7 adipokines (leptin, adiponectin, resistin, visfatin, osteopontin, omentin and chemerin) and found that high levels of visfatin to be associated with lesser functional impairment for patients with similar levels of resistin.

Presle et al. [15] demonstrated that the high serum concentration of visfatin may be due to increased permeability of the inflamed synovial membrane. They also suggested that the synovium, infrapatellar fat pad and osteophytes were important sources of visfatin in the joint.

Brentano et al. [16] found that concentrations of visfatin in serum correlated with the degree of inflammation and functional impairment in patients with $\mathrm{OA}$ according to Lequesne's score. They also found visfatin itself promotes inflammatory and destructive processes in the joints of patients with OA.

Wei-ping Chen et al. [4], analyzed the gene expression of visfatin in articular cartilage. Their data indicated that visfatin expression was significantly higher in cartilage in patients with OA than in donors. This suggests that visfatin might be involved in the pathophysiology of OA.

Our results are consistent with another study reported by Gosset et al., [17] who showed positive correlation between visfatin level to the degree of inflammation and functional impairment of $\mathrm{OA}$, by estimating concentration of visfatin in serum, and explained that was due to the potent proinflammatory and pro-degradative effects of visfatin in the progression of OA [17].

Our study's limitations were: the small sample number of patients as they were recruited from one area, Benha university hospitals and consequently the number of male patients that involved in the study. Another limitation was the unavailability of measurement of visfatin level in synovial fluid because of refusal of the majority of our patients to be aspirated.

\section{Conclusion:}

We concluded that serum visfatin may be involved in the pathogenesis of Rheumatoid arthritis and Osteoarthritis diseases, and may act as a good predictor for activity and severity of RA, as well as the severity of OA . 


\section{Recommendations:}

The study should be conducted on large sample number and include more male gender.

The relation between Visfatin and DMARD in RA patients should be studied.

\section{Conflict of interest:}

None of the contributors declared any conflict of interest.

\section{References:}

1) Joan Calvet , Cristóbal Orellana , Antoni Berenguer Llergo , Juan J Chillarón , Marta Larrosa (2016): Synovial fluid adipokines are associated with clinical severity in knee osteoarthritis: a crosssectional study in female.; Arthritis research \& therapy 18:207

2) Marie-Charlotte Laiguillon, Xavier Houard, Carole Bougault, Marjolaine Gosset , Geoffroy Nourissat, Alain Sautet, et al., (2014) : Expression and function of visfatin (Nampt), an adipokineenzyme involved in inflammatory pathways of osteoarthritis. Arthritis Research \& Therapy16:R38

3) Wei-ping Chen, Jia-peng Bao, Jie Feng , Peng-fei Hu , Zhong-li Shi and Li-dong Wu (2018): Increased serum concentrations of visfatin and its production by different joint tissues in patients with osteoarthritis. Clin Chem Lab Med;48(8):11411145

4) Joan Calvet, Cristóbal Orellana , Antoni Berenguer Llergo, Juan J Chillarón, Marta Larrosa (2016): Synovial fluid adipokines are associated with clinical severity in knee osteoarthritis: a crosssectional study in female.; Arthritis research \& therapy 18:207

5) Cooles F.A. and Isaacs J.D. (2011): Pathophysiology of rheumatoid arthritis. Curr. Opin. Rheumatol.; 23:233-240.

6) Altman,R.,Alarcon, G., Appelrouth, D., Bloch,D.,Borenstein,D., Brandt.(1986): Clinical/radiographic symptomatic criteria osteoarthritis .Arthritis Rheum, 34, 505.

7) Aletaha D1, Neogi T, Silman AJ.(2010) : 2010 Rheumatoid arthritis classification criteria: an American College of Rheumatology/European League Against Rheumatism collaborative initiative. Arthritis Rheum.;62(9):2569-81

8) Visser K. (2015): Optimal dosage and route of administration of metho-trexate in rheumatoid arthritis. Ann Rheum Dis; 68:1094-1099.

9) Elis Carolina , Flávia Troncon Rosa , Andréa Simão and Isaias Dich (2018): Adipokines in rheumatoid arthritis., Advances in Rheumatology 58:25 p. $1-10$.

10) Hashizume $M$ and Mihara M, (2011): The Roles of Interleukin-6 in the Pathogenesis of Rheumatoid Arthritis, Volume 11 , Article ID 765624,8 pages

11) Alkady EAM , Ahmed HM , Tag L and Abdou MA.( 2011): Adiponectin, Resistin and Visfatin in Serum und Gelenkflüssigkeit bei Patienten mit rheumatoider Arthritis. Z Rheumatol.;70(7):602-8

12) El-Hini SH, Mohamed FI, Hassan AA, Ali F, Mahmoud A and Ibraheem HM.( 2013): Visfatin and adiponectin as novel markers for evaluation of metabolic disturbance in recently diagnosed rheumatoid arthritis patients. Rheumatol Int.;33(9):2283-9.

13) Sglunda $\mathrm{O}$, Mann H, Hulejová H, Kuklová M , Pecha O , Pleštilová L(2014): Decreased 
circulating visfatin is associated with improved disease activity in early rheumatoid arthritis: data from the PERAC cohort. PLoS One.;9(7):1-5.

14) Joan Calvet, Cristóbal Orellana , Antoni Berenguer Llergo , Juan J Chillarón and Marta Larrosa (2016): Synovial fluid adipokines are associated with clinical severity in knee osteoarthritis: a crosssectional study in female.; Arthritis research \& therapy 18:207

15) Presle N, Pottie P, Dumond H, Guillaume C, Lapicque F, Pallu S (2016): Differential distribution of adipokines between serum and synovial fluid in patients with osteoarthritis. Contribution of joint tissues to their articular production. Osteo arthr Cartilage ;14:690-5.

16) Brentano F, Schorr O, Ospelt C (2007): Pre-B cell colony-enhancing factor/visfatin, a new marker of inflammation in rheumatoid arthritis with proinflammatory and matrixdegrading activities. Arthritis Rheum; 56: 2829 - 2839.

17) Gosset M, Berenbaum F, Salvat C, Sautet A , Pigenet A and Tahiri K (2018) :Crucial role of visfatin/pre-B cell colony-enhancing factor in matrix degradation and prostaglandin E2 synthesis in chondrocytes: possible influence on osteoarthritis. Arthritis Rheum 58(5):1399-1409

To cite this article: Dalia Allam, Abd El Wahab Shams El Din, Ahmed El Shambaky, Marwa Yehia. Visfatin in Association with Severity and Activity of Primary Osteoarthritis and Rheumatoid Arthritis. BMFJ 2021;38(1): 113-124. DOI: 10.21608/bmfj.2020.44849.1322 